\title{
Increased spatial salience in the social Simon task: A response-coding account of spatial compatibility effects
}

\author{
Kerstin Dittrich • Annelie Rothe • \\ Karl Christoph Klauer
}

Published online: 20 April 2012

(C) Psychonomic Society, Inc. 2012

\begin{abstract}
A spatial compatibility effect (SCE) is typically observed in forced two-choice tasks in which a spatially defined response (e.g., pressing a left vs. a right key) has to be executed to a nonspatial feature of a stimulus (e.g., discriminating red from green) that is additionally connoted by a spatial feature (e.g., the stimulus points to the left or the right). Responses are faster and more accurate when the response side and the spatial stimulus feature are compatible than when they are incompatible. Previous research has demonstrated that SCEs are diminished when stimuli from only one response category are responded to in individual go/no-go tasks, whereas SCEs reemerge when two participants work jointly on two complementary, individual go/no-go tasks in a joint go/no-go task setting. This social Simon effect has been considered evidence for shared task representations. We show that SCEs emerge in individual go/no-go tasks when the spatial dimension is made more salient, whereas SCEs are eliminated in joint go/no-go tasks when the spatial dimension is made less salient. These findings are consistent with an account of social Simon effects in terms of spatial response coding, whereas they are inconsistent with an account of shared task representations. The relevance of social factors for spatial response coding is discussed.
\end{abstract}

Keywords Simon effect - Co-representation - Joint action · Spatial compatibility task $\cdot$ Response coding

One of the most extensively studied interference paradigms is the spatial compatibility task (often called a Simon-like task: Simon, 1990; see Lu \& Proctor, 1995, for an overview). In a typical spatial compatibility task, participants respond to a

K. Dittrich $(\bowtie) \cdot$ A. Rothe $\cdot$ K. C. Klauer

Institut für Psychologie, Albert-Ludwigs-Universität Freiburg,

79085 Freiburg, Germany

e-mail: dittrich@psychologie.uni-freiburg.de dichotomous stimulus feature (e.g., a stimulus that can either be green or red) using two spatially arranged response keys (e.g., a right key and a left key). A task-irrelevant spatial stimulus dimension is induced by changing the positions of the presented stimuli (e.g., the stimulus appears on either the right or the left side of the computer screen). A typical finding is that responses are faster and more accurate when the spatial features of the stimulus and of the required response correspond (compatible trial) than when they do not correspond (incompatible trial). This spatial compatibility effect (SCE) is primarily explained in terms of response interference at the response-selection stage. The dimensionaloverlap model (Kornblum, Hasbroucq, \& Osman, 1990) provides a well-known theoretical framework to explain the underlying processes of SCEs. SCEs in this model are explained by the dimensional overlap (categorical similarity) of the stimulus and the response set. Given this overlap, the spatial information of the stimulus automatically activates a corresponding response that interferes with the required response in incompatible trials, leading to longer reactions times and higher error rates, although the spatial information of the stimulus is task-irrelevant in the spatial compatibility task.

Typically, spatial compatibility tasks consist of forced two-choice tasks in which participants are requested to respond to each presented stimulus. However, SCEs have also been examined in individual go/no-go tasks, in which participants only respond to certain stimuli in go trials, while they refrain from responding to other stimuli in no-go trials (Hommel, 1996; Shiu \& Kornblum, 1999; see Liepelt, Wenke, Fischer, \& Prinz, 2011, for a sequential trial-by-trial analysis of SCEs in individual go/no-go tasks). Small SCEs were even found in individual go/no-go tasks, but as Hommel pointed out, both response alternatives have to be activated at least internally in order to find SCEs in those kinds of tasks. 
Confirming this assumption, he found SCEs in individual go/ no-go tasks when two responses, not one, were held in readiness (i.e., one vs. two response keys had to be pressed during the experiment). The absence of SCEs in individual go/no-go tasks when only one response is activated can also be explained in terms of the dimensional-overlap model (Kornblum et al., 1990): If only one response key is instructed to be pressed during the experiment, the response dimension has no spatial connotation, and thus participants might not perceive and code their responses spatially; the response dimension no longer overlaps with the task-irrelevant spatial stimulus dimension, and SCEs typically fail to occur.

Recently, these basic findings of SCEs have been extended by studies on shared task representations (Sebanz, Knoblich, \& Prinz, 2003, 2005; Sebanz, Knoblich, Prinz, \& Wascher, 2006; Tsai, Kuo, Jing, Hung, \& Tzeng, 2006). This line of research is based on the assumption that people share action representations and coordinate their actions with those of others to achieve common goals (Clark, 1996). In a first study, Sebanz et al. (2003) presented pictures of a human hand that pointed either to the left, the middle, or the right-hand side of the computer screen and that had either a red or a green ring on one finger. Participants were asked to respond to the color of the ring and were instructed to ignore the pointing direction of the hand (task-irrelevant spatial stimulus feature). In a forced two-choice task, participants responded to one color with a right buttonpress and to the other color with a left buttonpress; as expected, an SCE was observed. In an individual go/no-go task, however, participants responded to only one color using a single response button; in this case, only small, nonsignificant SCEs were found. This result is in line with the assumption that the single-response option shares no dimensional overlap with the task-irrelevant stimulus dimension; thus, the prerequisite for spatial compatibility to emerge was missing in this task setting (see the explanation above). Interestingly, when the two complementary response alternatives of the regular forced two-choice task were distributed across two participants in a joint go/no-go task (a so-called social Simon task), SCEs prevailed for each individual (i.e., a social Simon effect emerged), although the task itself resembled a go/no-go task for each individual.

The presence of SCEs in joint but not in individual go/ no-go tasks has been interpreted as evidence for a shared task representation that is based on a common coding of perceived events and planned actions in joint go/no-go task settings (Greenwald, 1970; Hommel, Müsseler, Aschersleben, \& Prinz, 2001; Prinz, 1997); that is, the action that one performs and the action that one observes are assumed to be represented in a functionally equivalent way. Therefore, sharing a spatial compatibility task should be quite similar to performing the task on one's own: When observing the coactor's responses to actual "no-go" stimuli in a joint go/no-go task, a task representation is induced that is comparable to the task representation of an individual solving a forced two-choice task (Sebanz et al., 2003, 2005).

The conclusiveness of this interpretation rests on the assumption that only the social context differs between the individual and the joint go/no-go conditions, and thus that the social context is the cause of SCEs in joint go/no-go tasks: Only if the individual and joint go/no-go tasks are assumed to be functionally equivalent can differences in the effects of the two tasks be ascribed to the social context and to shared task representations. However, sitting next to a coactor in front of a computer screen also changes the experimental setting in other ways. It might be possible that the social setting renders the horizontal spatial response dimension more salient than the individual go/no-go task setting does. This might directly influence the way that participants code their responses: In joint go/no-go tasks, the presence of a coacting person allows participants to code their responses as being either the left response or the right response, depending on the seating position of the coacting person. In terms of the dimensional-overlap model, the response dimension thus might again overlap with the task-irrelevant spatial stimulus dimension, leading to interference effects in the case of incompatible trials. This raises the possibility that SCEs in joint go/no-go tasks do not reflect shared task representations. Instead, joint SCEs might go back to the increased salience of the spatial response dimension in joint go/no-go tasks as compared to individual go/no-go tasks, which would allow participants to spatially code their responses relative to coacting participants.

At first glance, a spatial-response-coding account of joint SCEs might stand in opposition to several recent studies that have demonstrated a modulation of joint SCEs via social factors (e.g., Hommel, Colzato, \& van den Wildenberg, 2009; Müller, Brass, et al., 2011; Müller, Kühn, et al., 2011; Tsai \& Brass, 2007). By reinterpreting these findings, we predict that social factors might modulate spatial response coding instead of shared task representations; that is, social factors might enhance the spatial interpretation of a situation. Notably, joint SCEs are small or absent when the other person is only present, but not actively participating (Sebanz et al., 2003). This suggests that the other person has to be perceived as a part of the task in order to provide a spatial reference point for one's own responses (see also Guagnano, Rusconi, \& Umiltà, 2010, for a similar line of argument). In our General Discussion, we will discuss in more detail how a spatialresponse-coding account of joint SCEs can be integrated into the existing literature of social factors in joint spatial compatibility tasks.

Evidence for the assumption that a spatial component might be responsible for joint SCEs to occur was recently found by Guagnano et al. (2010). In their first experiment, they eliminated the turn-taking character of the joint go/no- 
go task by presenting two stimuli simultaneously in most of the trials, one from each response category. It was hypothesized that this procedure would lead both participants to perceive their respective tasks as independent from one another. For that reason, this procedure should eliminate the necessity of action coordination between participants, because both participants would no longer share a common goal in the task. Even though this procedure was expected to eliminate shared representations of the task, a small SCE was nonetheless found. Additionally, Guagnano et al. demonstrated in a similar task that joint SCEs were only observed when participants sat within arm's reach, but not when they sat farther apart. Guagnano et al. concluded that coacting with another person sitting to the left or the right of oneself provides a spatial reference point that might be responsible for the emergence of SCEs in joint go/no-go tasks. More specifically, they argued that actors spatially code their responses when a spatial reference point (a coacting person sitting within arm's reach) is given: An actor sitting on the left side of a coacting person is assumed to code his or her own response as a left response relative to the coactor, while the right-sitting coacting person is assumed to code his or her own response as a right response. In addition to the results reported by Guagnano et al., Dolk et al. (2011) provided evidence for a nonsocial explanation of the social Simon effect. In their first experiment, the authors attempted to moderate the joint SCE by manipulating the sense of ownership of the coactor's hand by inducing a rubber hand illusion (see Botvinick \& Cohen, 1998). Interestingly, joint SCEs decreased rather than increased with facilitated integration of the coactor's actions. Furthermore, SCEs were even observed in a go/no-go condition in which no coactor was present but the inactive hand of the participant was stimulated.

By eliminating the turn-taking character of the spatial compatibility task (Guagnano et al., 2010) or by inducing the rubber hand illusion as an additional influencing factor (Dolk et al., 2011), evidence was provided that the spatial component of the task leads to joint SCEs rather than to shared task representations. Still, the underlying processes remained unclear. Although Guagnano et al. pointed out that the spatial reference point provided by the coacting individual is what allows participants to code their responses spatially, the researchers primarily manipulated the task on the stimulus dimension, but not systematically on the response dimension. Thus, the assumption of a spatial coding of responses was tested rather indirectly. Additionally, Guagnano et al. argued that the response codes in joint go/no-go tasks might act as a prime and facilitate responses in a joint go/nogo task. But this stands in contrast to recent results provided by Ferraro, Iani, Mariani, Milanese, and Rubichi (2011), who found that joint SCEs are composed of interference rather than of facilitation effects. Thus, the new interpretation of joint SCEs provided by Guagnano et al. was put in question by the very recent experiments of Ferraro et al.
The present research attempted to provide more direct evidence for spatial response coding as an alternative explanation of joint SCEs. If spatial coding of responses is indeed responsible for SCEs, it should be possible to switch SCEs on and off by increasing and decreasing, respectively, the salience of the spatial response dimension. We set out to show that manipulations of the spatial salience of the response dimension indeed determine whether or not SCEs prevail in individual go/no-go tasks (Exp. 1), as well as in joint go/no-go tasks (Exps. 2 and 3). Thus, we tested the role of spatial response coding for both joint and individual go/no-go tasks, addressing the two lines of Sebanz et al.'s (2003) original argument comprehensively: (1) no SCEs in individual go/nogo tasks and (2) SCEs in joint go/no-go tasks. To anticipate, this design not only provided evidence for the relevance of a spatial reference point for joint SCEs (Guagnano et al., 2010), but more generally, it provided evidence for the role of the salience of the spatial response dimension in allowing participants to spatially code their responses in different task settings. The result pattern also permitted us to rule out that shared task representations had caused the SCEs observed in joint go/no-go tasks. Our results were therefore in line with recent results demonstrating that even nonsocial factors are sufficient to induce SCEs in individual go/no-go tasks (Dolk et al., 2011).

\section{Experiment 1: Individual go/no-go tasks}

In Experiment 1, we addressed the first critical effect pattern that has served as a proof for shared task representations: the absence of SCEs in individual go/no-go tasks that nevertheless elicit SCEs when performed as forced two-choice tasks. According to our line of argument, the absence of SCEs in individual go/no-go tasks reflects the fact that only using a single response key does not emphasize the spatial connotation of this response key (e.g., being the left key vs. the right key) to the same extent as in forced two-choice tasks: There is thus no dimensional overlap of the response dimension and the stimulus dimension, and therefore no SCEs prevail in individual go/no-go tasks.

Participants either performed an individual go/no-go task or a two-alternative forced choice task; orthogonally, they responded either via response keys (we used the interior keys of two computer mice positioned to the left and right in front of the participants; the participants pressed the mouse keys with their index fingers) or via a left versus a right movement of a joystick. We expected the salience of the spatial response dimension to be high in forced twochoice tasks (the participants were aware that they had to execute a left and a right response), irrespective of the response mode, and thus we predicted SCEs in the forced two-choice tasks for both response modes.

It is important to note that SCEs should emerge even in the individual go/no-go task when the responses are 
delivered via joystick movements. This hypothesis is based on the following assumptions: The use of left versus right joystick movements to go stimuli endows these responses with an obtrusive spatial feature; that is, the spatial movement induces participants to code their response spatially (i.e., the movement is perceived and coded as being a left or a right response). According to the dimensional-overlap model (Kornblum et al., 1990), this spatial response coding causes the response dimension to overlap with the stimulus dimension, and even leads to SCEs in individual go/no-go tasks.

Replicating previous work (e.g., Sebanz et al., 2003), SCEs should not emerge when responses require keypresses in individual go/no-go tasks: Responses are not spatially coded, and thus the response dimension and the stimulus dimension do not overlap. See Fig. 1 for an illustration of the stimulus-response overlaps assumed in the different conditions.

Summing up, the main predictions are as follows: Inasmuch as SCEs depend on the salience of the spatial response dimension, (a) SCEs should be absent in the individual go/nogo response key group, whereas they should be present in the individual go/no-go joystick group. Consequently, the SCEs in both individual go/no-go conditions should differ significantly. In addition, (b) SCEs should be observed in both the forced two-choice response key and joystick groups.

\section{Method}

Participants A group of 70 right-handed University of Freiburg students (46 female and 24 male) with different majors participated for course credit or as paid volunteers; their mean age was 24 years $(S D=5.51)$, ranging from 18 to 44 years. All of the participants had normal or corrected-tonormal vision. They were randomly assigned to one of four experimental conditions defined by two between-participants factors: task (individual go/no-go vs. forced two-choice) and response device (joystick vs. response keys).
Materials and apparatus The stimuli from Tsai et al. (2006) were used: A white rectangle (subtending a solid angle of approximately $8.5^{\circ} \times 3.3^{\circ}$ in width and height, in terms of visual angle) containing three white, unfilled discs (a solid angle of approximately $1^{\circ}$ in radius and $0.5^{\circ}$ in distance between the discs, in terms of visual angle) was presented on a blue background in the center of a computer screen throughout an experimental block. The three white discs were positioned on the left half, in the middle, and on the right half of the rectangle. The targets were either a red or a green filled circle replacing one of the white discs in each trial. Responses were made by left versus right joystick movements or left versus right keypresses (we used the interior keys of two computer mice, see Voss, Leonhart, \& Stahl, 2007), depending on group membership. The computer mice were connected to the computer's parallel port and were positioned in front of the computer screen at a distance of $80 \mathrm{~cm}$ from each other (see Fig. 2); the joystick was connected to the computer's game port. The joystick movement triggered a measurement when the lever subtended an angle of approximately $25^{\circ}$, which was near the dead-stop position. Each participant sat approximately $60 \mathrm{~cm}$ away from a $48.3-\mathrm{cm}$ computer screen with a 100 $\mathrm{Hz}$ refresh rate.

Procedures The participants were tested in individual sessions that lasted around $30 \mathrm{~min}$. They performed two practice blocks of 60 trials each and four experimental blocks of 126 trials each. The first practice block served as a familiarization task; therefore, the timing in this block was slower than in the following practice and experimental blocks. Each trial started with the presentation of the white rectangle with three white discs for $400 \mathrm{~ms}$, followed by the target (i.e., a red or green circle replaced one of the three white discs). In the experimental blocks and in the second practice block (values for the first practice block are shown

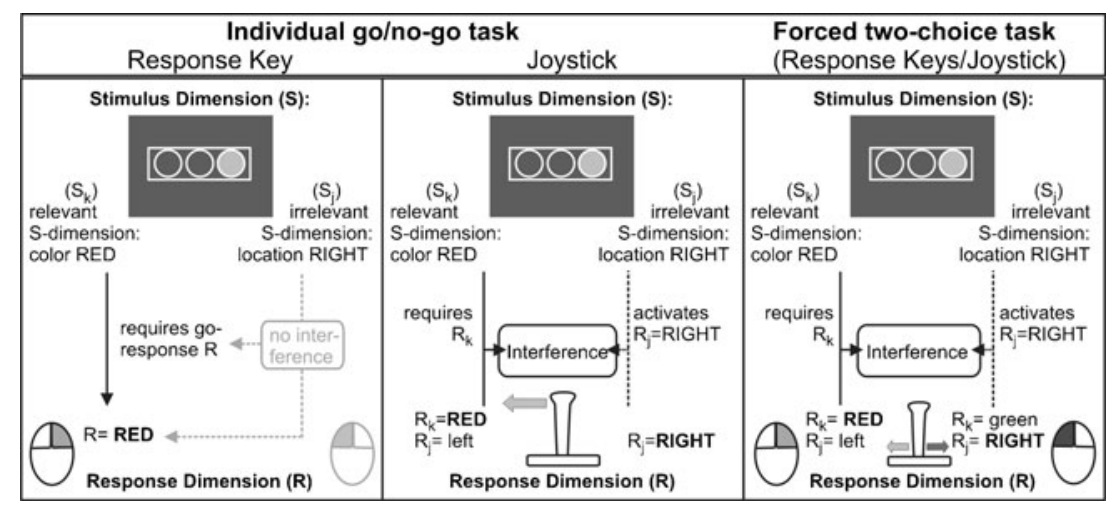

Fig. 1 Illustrations of the stimulus (S)-response (R) dimensions for incongruent trials in the individual go/no-go and forced two-choice tasks of Experiment 1 for joystick and response keys, in accordance with the dimensional-overlap model (Kornblum et al., 1990). $\mathrm{S}_{\mathrm{k}}=$ relevant stimulus dimension, $S_{j}=$ irrelevant stimulus dimension, $R_{k}=$ assigned/required response, $\mathrm{R}_{\mathrm{j}}=$ activated response. Responses have to be spatially coded in order to induce a dimensional overlap of the response dimension and the stimulus dimension. In Experiment 1, a spatial response coding is assumed to be induced by joystick movements 


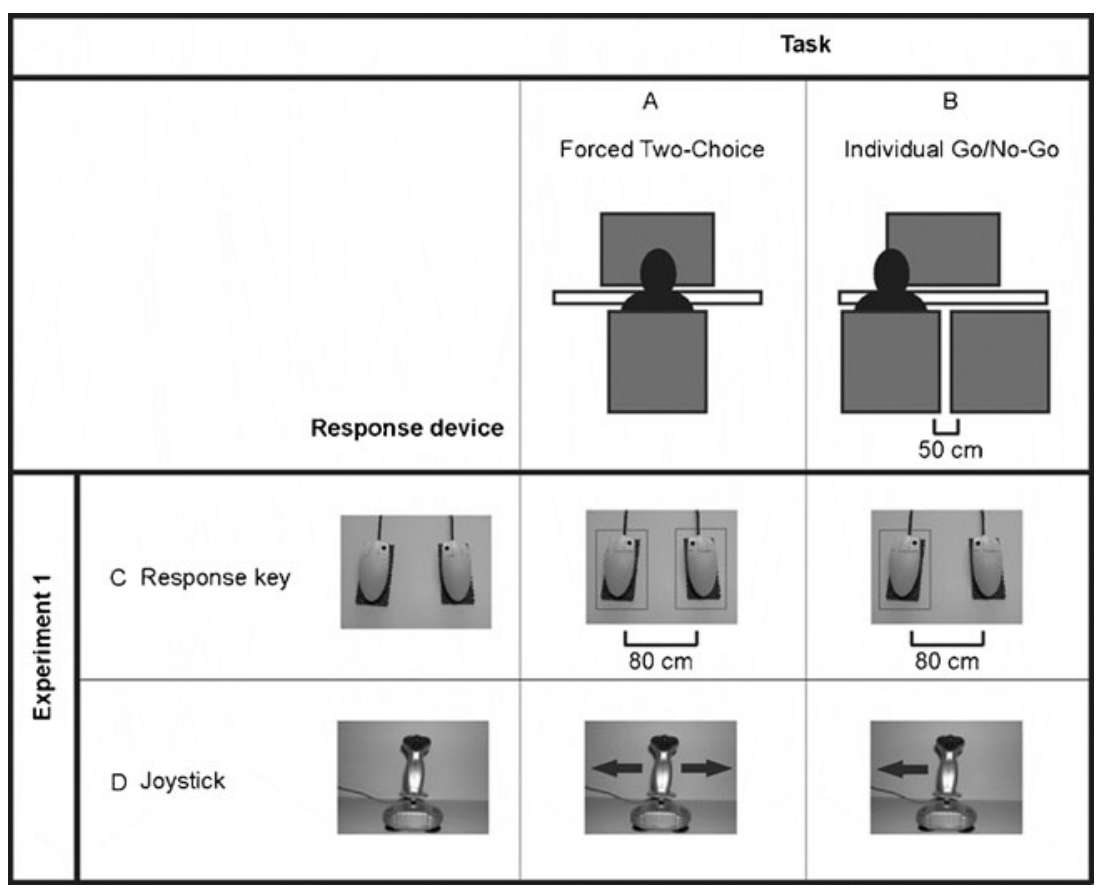

Fig. 2 Tasks, participants' seating alignments, and response devices used in Experiment 1. The participants performed either a forced twochoice task (a) or an individual go/no-go task (b). Orthogonally, they responded with either response keys (c) or joystick movements (d). Participants performing the forced two-choice task responded with both response keys or with left and right joystick movements (indicated by rectangles/arrows in the figure). Participants performing the

in parentheses), the target remained on screen until $150 \mathrm{~ms}$ $(3,000 \mathrm{~ms})$ had passed or a response was entered. Reaction times were measured from the beginning of the target display, and there was a time period of $600 \mathrm{~ms}(3,000 \mathrm{~ms})$ for responding. Responses exceeding this time limit were considered omissions. The intertrial interval was $500 \mathrm{~ms}$. Stimuli that did not require a response disappeared after $150 \mathrm{~ms}$ $(1,000 \mathrm{~ms})$, and the next trial started after $950 \mathrm{~ms}(500 \mathrm{~ms})$. Participants received error feedback for responses that were incorrect or too slow; either "Fehler!" (Error!) or "Zu langsam!" (Too slow!) was presented in the center of the screen for $500 \mathrm{~ms}$.

In the forced two-choice groups, the participants sat in the middle of the table in front of the computer screen. Half of them responded with joystick movements, the other half with keypresses. They responded to one color with a left joystick movement or by pressing the key of the left computer mouse and to the other color with a right joystick movement or by pressing the key of the right computer mouse. In the individual go/no-go groups, participants sat either on the left or the right side of the table in front of the computer screen with an empty chair beside them. They were instructed to only respond to one color with one specific joystick movement or by pressing the interior key of just one of the two computer mice. Response side was aligned with the seating arrangement: For example, participants sitting on the left responded individual go/no-go task either sat on the left side of the table and responded by pressing the key of the left computer mouse or by pushing the joystick to the left (as displayed in this figure) or sat on the right side of the table and either pressed the key of the right computer mouse or made a right joystick movement. Distance information is indicated in centimeters

by moving the joystick to the left or by pressing the key of the left mouse (as in the forced two-choice condition, both mice were positioned to the left and right in front of the computer screen). ${ }^{1}$ Fig. 2 depicts the experimental procedure of Experiment 1.

\section{Results}

The accuracy data (percentage correct scores of trials, without errors and omissions) were arcsine-transformed for the statistical analyses in this and the subsequent experiments, but the untransformed original percentage scale will be used here for ease of interpretation when mean values are reported in figures, tables, and the body of the text. For the individual go/no-go joystick condition, movements to the wrong side were counted as errors. These are part of the error rates reported in Experiment 1, appeared rarely, and were committed by only three participants. Excluding these three participants from the statistical analyses did not influence the result pattern.

\footnotetext{
${ }^{1}$ Note that the lateral seating position of participants in the individual go/no-go task was inspired by the experimental procedure of the seminal work by Sebanz et al. (2003), which we relied on as closely as possible (see also, e.g., Tsai et al., 2006, for a similar procedure).
} 
Response latencies below $150 \mathrm{~ms}$ and outliers were omitted from each individual's latency distribution; outliers were identified by Tukey's criterion (i.e., as latencies below the first quartile minus 1.5 times the interquartile range or above the third quartile plus 1.5 times the interquartile range; Clark-Carter, 2004, chap. 9). This led to the exclusion of $0.63 \%$ of the trials. The trials were coded as compatible (stimulus-response correspondence), incompatible (stimulusresponse noncorrespondence), and neutral (the imperative stimulus was presented in the middle), according to the spatial feature of the target and the required response. The mean response latencies and mean error rates for compatible, incompatible, and neutral trials are presented separately for the forced two-choice tasks and the individual go/no-go tasks in Table 1 in the Appendix. In Fig. 3, SCEs are presented as a function of task (individual go/no-go vs. forced two-choice) and response device (joystick vs. response key) for latencies (upper panel) and error rates (lower panel).
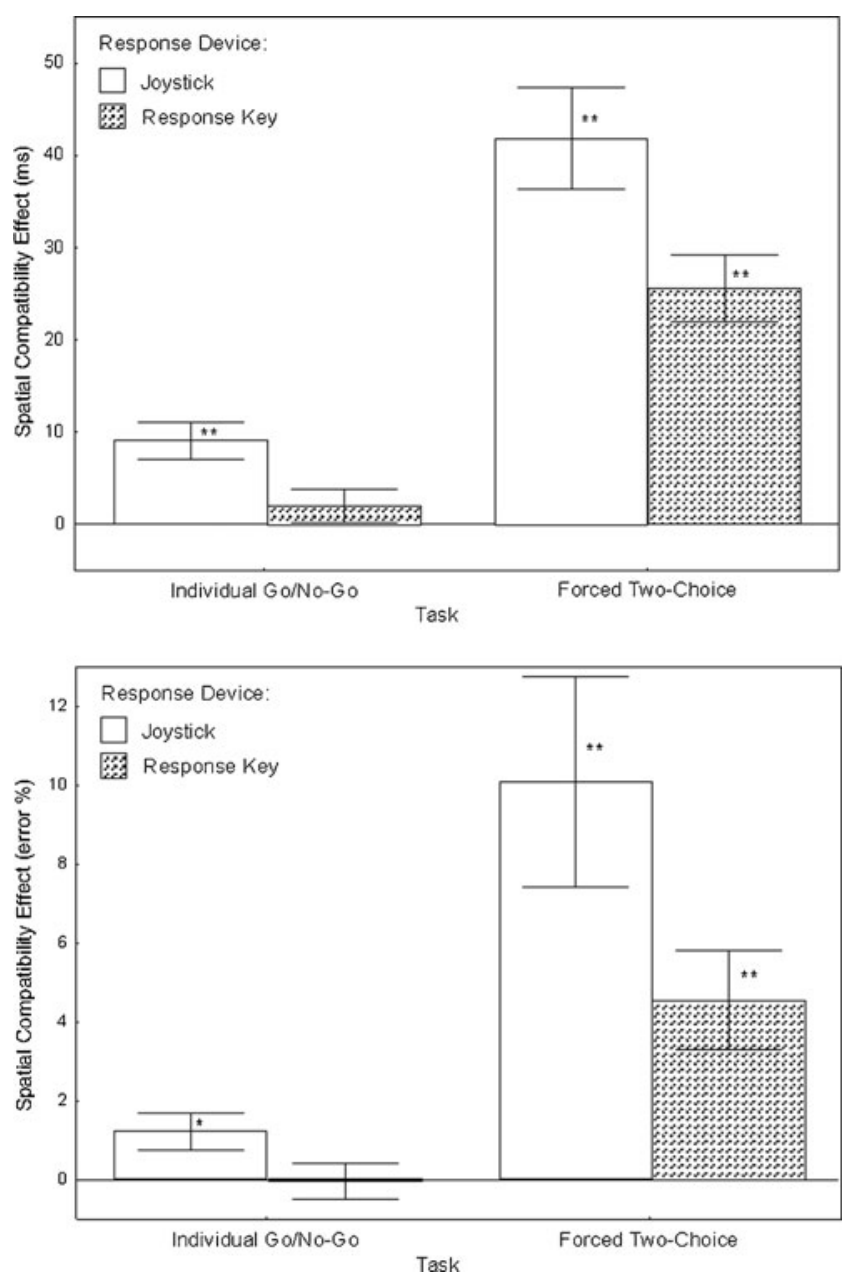

Fig. 3 Experiment 1: Spatial compatibility effects in the latency domain (upper panel) and the error domain (lower panel) as a function of task (individual go/no-go vs. forced two-choice) and response device (response key vs. joystick). Error bars show standard errors of the means. ${ }^{*} p<.05,{ }^{* *} p<.01$
The response latency and accuracy data were submitted to $3 \times 2 \times 2$ analyses of variance with the factors Compatibility (compatible vs. incompatible vs. neutral) $\times$ Response Device (joystick vs. response key) $\times$ Task (individual go/nogo vs. forced two-choice) and repeated measures on the first factor. Strong effects of compatibility for both response latencies, $F(2,132)=106.40, p<.01, \eta_{p}{ }^{2}=.62$, and the accuracy data, $F(2,132)=27.43, p<.01, \eta_{p}^{2}=.29$, revealed faster responses and fewer errors in compatible than in incompatible trials. As expected, the compatibility effects were larger in the forced two-choice conditions, reflected by a significant interaction of compatibility and task in response latencies, $F(2$, $132)=45.35, p<.01, \eta_{p}{ }^{2}=.41$, and in the accuracy data, $F(2$, $132)=10.74, p<.01, \eta_{p}^{2}=.14$. In addition, compatibility interacted with response device in the latency domain, $F(2$, $132)=8.36, p<.01, \eta_{p}{ }^{2}=.11$, reflecting larger SCEs for participants responding with the joystick; there was also a threeway interaction of compatibility, response device, and task in the latency domain, $F(2,132)=3.10, p<.05, \eta_{p}{ }^{2}=.05$ (see the $t$ tests below). Moreover, the analyses of variance revealed a main effect of response device: Faster responses and fewer errors occurred in the response key conditions than in the joystick conditions, $F(1,66)=91.98, p<$ $.01, \eta_{p}{ }^{2}=.58$, and $F(1,66)=12.78, p<.01, \eta_{p}{ }^{2}=.16$, respectively. The factor Task showed a further main effect: Responses were faster and fewer errors were made in the individual go/no-go tasks than in the forced two-choice tasks, $F(1,66)=21.20, p<.01, \eta_{p}{ }^{2}=.24$, and $F(1,66)=$ $80.91, p<.01, \eta_{p}^{2}=.55$, respectively.

Follow-up $t$ tests were conducted to test our predictions that (a) SCEs would appear in the individual go/no-go joystick group but not in the individual go/no-go response key group and (b) SCEs would appear in both the forced two-choice response key and joystick groups. (a) As predicted, SCEs were significant in the individual go/no-go joystick group both for response latencies, $t(23)=4.42, p=$ .01 , and the accuracy data, $t(23)=-2.43, p=.02$. In the individual go/no-go response key group, SCEs were not significant for either response latencies $(t=1.16)$ or the accuracy data $(t=-0.11)$. As expected, response interference was significantly larger in the individual go/no-go joystick group than in the individual go/no-go response key group, $t(46)=2.59, p=.01$, whereas this difference was not significant for the accuracy data, $t=1.6$. Note that the error rates in the individual go/no-go tasks were small (see Table 1 in the Appendix) but comparable to the small error rates reported in other studies examining individual or joint SCEs (e.g., Sebanz et al., 2003, Exp. 1: 1.6\% in the individual go/no-go task). This might explain why the difference of SCEs in the error rates of both individual go/no-go conditions failed to reach significance. (b) As predicted, significant SCEs were observed in both the forced two-choice joystick group $[t(10)=7.58, p<.01$, and $t(10)=-4.15, p<.01$, respectively 
for the latency and accuracy domains] and the forced twochoice response key group $[t(10)=7.09, p<.01$, and $t(10)=-3.68, p<.01$, respectively for the latency and accuracy domains]. Response interference was significantly larger in the forced two-choice joystick group than in the forced two-choice response key group, $t(20)=2.48, p=.02$; this difference was not significant in the accuracy domain, $t=1.2$.

\section{Discussion}

Confirming previous findings, no SCE was observed in the individual go/no-go task when the participants responded with response keys. However, a significant SCE emerged in the individual go/no-go condition when the participants responded with a joystick. Moreover, SCEs were significantly larger in the individual go/no-go joystick group than in the individual go/no-go response key group for response latencies. Interference effects were observed in the forced two-choice tasks for both joystick and key responses, revealing robust baseline effects.

The manual movement of the joystick in one direction highlighted the spatial response dimension sufficiently; that is, the spatial movement of the joystick to the left or the right side induced participants to perceive and code their responses as either left or right. Now that responses were spatially coded, the response dimension overlapped with the stimulus dimension: The response (movement of the joystick) clearly interfered with the location of the stimulus when the response direction did not correspond to the stimulus position. Consequently, Experiment 1 showed that it is possible to induce SCEs even in individual go/no-go tasks by increasing the salience of the spatial response dimension. At the same time, we replicated the absence of SCEs in individual go/nogo tasks under conditions of low salience of the spatial response dimension (e.g., Sebanz et al., 2003). In line with the dimensional-overlap model (Kornblum et al., 1990), we assumed that there was no overlap between the response dimension and the stimulus dimension in this condition (see Fig. 1 for a schematic depiction of the assumed processes in the different conditions).

As was noted in the Results section, three of the participants made movements to the wrong side in the individual go/no-go joystick condition. Although these errors occurred very rarely (in only a total of nine trials), they might still suggest that the difference between the individual go/no-go joystick and response key data was due to the mere availability of a second, task-irrelevant response option in the joystick condition. However, this explanation seems unlikely. Hommel (1996) demonstrated that the mere availability of task-irrelevant responses did not influence SCEs. In his second experiment, he asked participants to either press two different response buttons (two-response-key condition) or only one button (one-response-key condition) when responding to two different stimuli in a spatial reaction time task. In the one-response condition, participants placed both index fingers on the corresponding keys, while one key was task-irrelevant and remained unused in this condition. Overall, SCEs were only found in the two-response condition; merely placing an index finger on a task-irrelevant response key did not lead to reliable SCEs. In his fifth experiment, the participants responded with either one of two spatially arranged keys and switched from one response to the other between blocks; this procedure had produced reliable SCEs in previous experiments. Hommel administered two conditions: In one, participants placed two fingers on two active response keys, and in the other, the currently invalid key was made inactive by covering it with a plastic cap. In this condition, the unused finger could not press the capped key; thus, no inhibition of a keypressing response was required. The results revealed an SCE that occurred independently from the cap condition. Notably, the effect was even descriptively larger in the cap than in the no-cap condition. Hommel concluded that "placing the unused response finger on an active response key or a rigid plastic cap does not change the size of correspondence effects, thus ruling out a possible explanation of at least some of the present findings in terms of response inhibition processes" ( $p$. 565). It should be noted that the experiments reported by Hommel were procedurally different from our Experiment 1. Comparisons between the experiments should therefore be drawn cautiously. Nevertheless, Hommel's results provide evidence against the assumption that the mere physical availability of a task-irrelevant response option led to the SCE observed in the individual go/no-go joystick condition of our Experiment 1.

While Experiment 1 provided evidence that increased spatial salience can induce SCEs in individual go/no-go tasks, the remaining open question is whether the social setting used in previous joint go/no-go tasks could increase the spatial salience of the response dimension similarly to the use of joystick movements. This was tested in Experiment 2 .

\section{Experiment 2: Joint go/no-go tasks}

We assume that the SCEs found in joint go/no-go tasks may not be caused by the social context per se, but instead by the salience of the spatial response dimension. As Experiment 1 demonstrated, emphasizing the spatial response dimension induces SCEs even in individual go/no-go tasks. Hence, the spatial salience of the response dimension by itself may be responsible for the reemergence of SCEs in joint go/ no-go tasks - if and when the social setting emphasizes the spatial response dimension relative to individual go/ no-go tasks. 
The social context may lead to this effect because observing a coactor seated to the left or to the right emphasizes the left-right spatial feature of one's own responses. If so, SCEs should be eliminated even in joint go/no-go tasks if the spatial arrangements of the stimuli and the response keys are switched from a horizontal left-right arrangement to a vertical up-down arrangement. Although participants might still think of their responses in terms of left and right due to the social context, this would not be expected to interfere with the spatial up-down feature of the stimuli. That is, the left-right seating arrangement should not emphasize the vertical spatial dimension: No external vertical cue (e.g., another person or a vertical joystick movement) is given that would allow participants to code their responses as up or down responses; thus, no stimulus-response overlap would be expected to occur (see Kornblum et al., 1990). The task typically used in joint go/no-go tasks (horizontally arranged stimuli; e.g., Sebanz et al., 2003) differs with regard to this point: Coding responses as left or right along with the leftright seating arrangement provokes a stimulus-response overlap of the left-right stimulus and the response features (see also Fig. 1; while in Exp. 1 joystick movements were assumed to induce a spatial response coding, in Exp. 2 the coacting participant was assumed to serve as a spatial reference for one's own response, inducing spatial response coding in Exp. 2).

In contrast, according to the account in terms of shared task representations, the joint go/no-go task should be functionally equivalent to a forced two-choice task (e.g., Sebanz et al., 2003, 2005), irrespective of the horizontal-versusvertical arrangement of the stimuli and response keys. The prediction would therefore be that SCEs would be observed for both spatial arrangements.

Two versions of each task were used in Experiment 2. The first version was identical to the task in Experiment 1 . We term this task version the horizontal task version. In the second version, the stimulus arrangement was rotated by $90^{\circ}$, so that the stimuli were presented at the top, at the bottom, or in the middle of an upright rectangle, and the response keys were arranged vertically on a response pad. ${ }^{2}$ We term this the vertical task

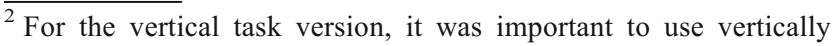
arranged response keys. Otherwise, there would be no possibility of a stimulus-response overlap, and thus no SCEs would be expected to prevail in either the forced two-choice task or the joint go/no-go task. Note that the vertical arrangement of the response keys was not expected to highlight the vertical response dimension to such an extent that joint SCEs would occur in this task version. This assumption is based on the observation of missing SCEs in individual go/no-go tasks (see, e.g., Exp. 1): Left- and right-positioned response keys do not highlight the horizontal response dimension to such an extent that SCEs prevail in individual go/no-go conditions.
}

version. Again, we implemented forced two-choice conditions for both spatial arrangements in order to ascertain that SCEs were strong in the horizontal as well as in the vertical version of the forced two-choice task. Task version (horizontal vs. vertical) and task (forced twochoice vs. joint go/no-go) were orthogonally crossed between participants. In the joint go/no-go groups, two participants sat beside each other; one participant had to respond to red stimuli, and the other participant had to respond to green stimuli. In the horizontal task version, the seating alignment (right vs. left side) corresponded to the horizontal stimulus and response key arrangements (right vs. left key). In the vertical task version, the participants' seating alignment (right vs. left side) corresponded with neither the vertical stimulus arrangement nor the vertical response key arrangement (upper vs. lower key). See Fig. 4 for a depiction of the stimulus arrangement, seating alignment, and response devices of Experiment 2.

To summarize the main predictions: Inasmuch as SCEs depend on the salience of the spatial response dimension, (a) SCEs should be absent in the vertical joint go/no-go task, since the seating alignment did not emphasize the vertical spatial dimension (and participants were not expected to code their responses as up or down responses), whereas in the horizontal joint go/no-go task, we expected to find SCEs. In addition, (b) SCEs should be observed in both the vertical and horizontal forced two-choice tasks. This pattern of predictions is inconsistent with an account in terms of shared task representations, according to which, if the vertical forced two-choice task induces SCEs, SCEs should also emerge in the joint go/no-go vertical task.

\section{Method}

Participants A group of 144 right-handed University of Freiburg students with different majors participated for course credit or as paid volunteers; their mean age was 23 $(S D=4.1)$, ranging from 18 to 51 years. All participants had normal or corrected-to-normal vision and were randomly assigned to one of four experimental conditions defined by task version (horizontal vs. vertical) and task (forced two-choice vs. joint go/no-go). The forced two-choice conditions comprised 48 participants, and the joint conditions included 48 pairs of participants. Two pairs of participants in the joint go/no-go group were excluded from the analyses because they responded to $36 \%$ and $41 \%$ of the no-go stimuli.

Materials and procedures The materials and procedures were identical to those of Experiment 1, except for the following differences. In the horizontal task, the stimuli 
Fig. 4 Stimulus arrangements, participants' seating alignments, and response devices used in Experiment 2. The participants performed either a forced two-choice task (a) or a joint go/no-go task (b). Orthogonally, they performed either a horizontal task version (c) or a vertical task version (d). Participants performing the horizontal task version responded with horizontally oriented response keys, and participants performing the vertical task version responded with vertically oriented response keys. Distance information is indicated in centimeters

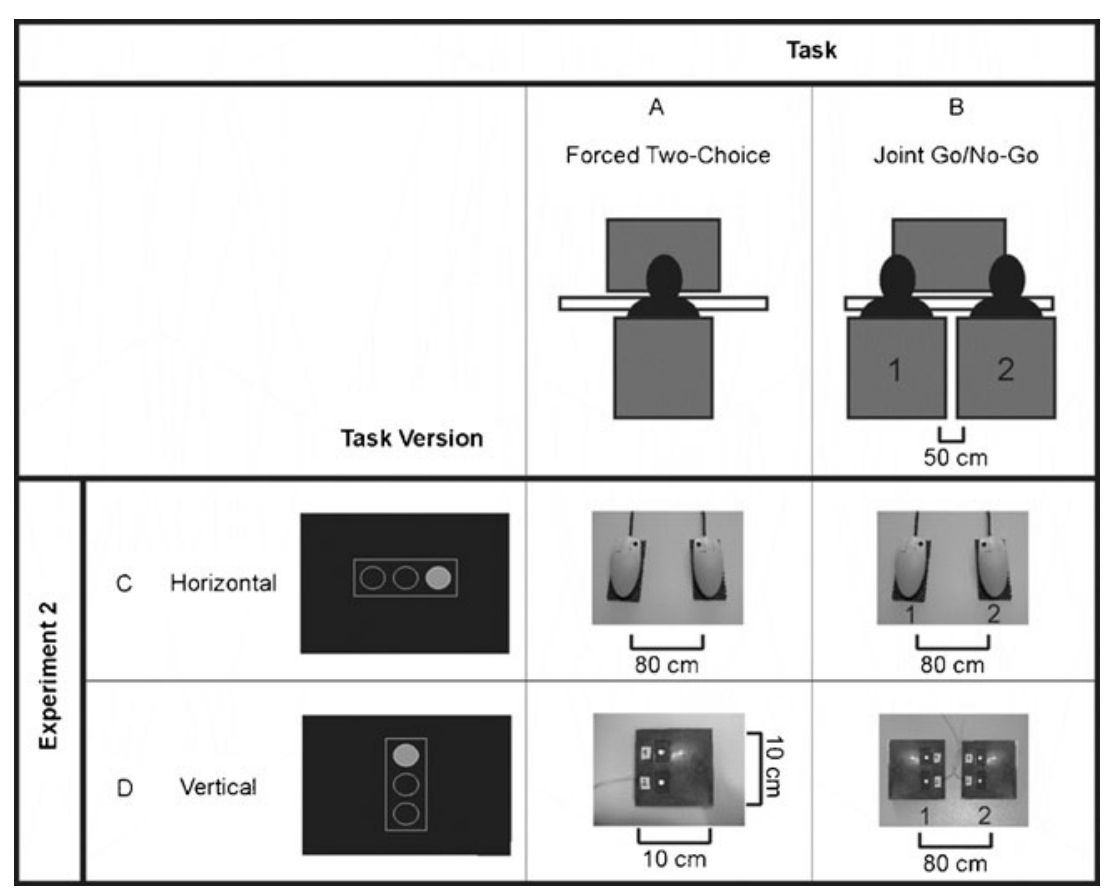

were identical to those of Experiment 1, and the participants responded with the interior keys of two computer mice positioned to the right and left of the computer screen at a distance of $80 \mathrm{~cm}$ from each other. Whether the right response key had to be pressed for green or red circles was counterbalanced. In the horizontal joint go/no-go task, one participant was seated to the right and responded with the right key, and the other participant was seated to the left and responded with the left key. Chairs were placed to the left and right in front of the computer screen at a distance of $50 \mathrm{~cm}$ away from each other. In the horizontal forced two-choice task, the participants sat in the middle in front of the computer screen and operated both response keys.

For the vertical task version, the stimulus arrangement was rotated by $90^{\circ}$. To respond, the participants used vertically arranged keys on $10 \times 10 \mathrm{~cm}$ response pads. In the vertical joint go/no-go task, two response pads were used and were positioned to the right and left sides of the PC screen at a distance of $80 \mathrm{~cm}$ from each other. The participant sitting on the right operated one key (e.g., the upper key) of the right response pad, whereas the other participant, sitting on the left, pressed the complementary key (e.g., the lower key) of the left response pad. Whether the right- or left-situated participant pressed the lower or the upper key and which color was mapped to each response key assignment was counterbalanced. In the vertical forced two-choice task, participants used a single response pad with two vertically arranged response keys installed in the middle in front of the PC screen, with either the upper or lower key assigned to red or to green stimuli. Whether participants were to press the lower or the upper key with their right or left index finger was counterbalanced. The stimulus arrangements, seating alignments, and response alignments are depicted in Fig. 4.

As has previously been shown in social cognition research, the instruction itself has the potential to influence how participants code their responses (e.g., Dittrich \& Klauer, 2012; Eder \& Rothermund, 2008). To ensure that the instructions did not bias participants' response codings, words like "right" and "left" or "upper" and "lower" were not mentioned. Instead, numbers (e.g., Participant 1 and Participant 2, Response Key 1 and Response Key 2) were used. In all conditions, participants received error feedback after each trial, as described in Experiment 1. In the joint go/no-go tasks, the feedback appeared on the side of the participant who had made a false response. Reaction times were measured until the "go participant" pressed the response key (if it was faster than $600 \mathrm{~ms}$ ). If the "no-go participant" erroneously responded first, the go participant still had the chance to respond. These trials were considered errors, and the no-go participant saw the error feedback on his or her side of the computer screen.

\section{Results}

Response latencies were preprocessed as in Experiment 1, which excluded $3.23 \%$ of the trials. The mean response latencies and mean error rates for compatible, incompatible, and neutral trials are presented in Table 2 in the Appendix, 
separately for the forced two-choice tasks and the joint go/ no-go tasks. In Fig. 5, SCEs are presented as a function of task version (horizontal vs. vertical) and task (joint go/no-go vs. forced two-choice) for latencies (upper panel) and error rates (lower panel).

The response latencies and accuracy data were submitted to analyses of variance with the factors Compatibility (compatible vs. incompatible vs. neutral) $\times$ Task Version (horizontal vs. vertical) $\times$ Task (joint go/no-go vs. forced two-choice) and repeated measures on the first factor. ${ }^{3}$ These analyses revealed main effects of compatibility in the latency domain, $F(2,180)=189.34, p<.01, \eta_{p}{ }^{2}=.68$, and in the accuracy domain, $F(2,180)=67.52, p<.01, \eta_{p}{ }^{2}=.43$, reflecting faster responses and fewer errors in compatible than in incompatible trials. Overall, compatibility effects were larger in the forced two-choice tasks than in the joint go/no-go tasks, as indicated by a significant interaction of compatibility and task for response latencies, $F(2,180)=96.30, p<.01, \eta_{p}{ }^{2}=.52$, and the accuracy data, $F(2,180)=55.12, p<.01, \eta_{p}{ }^{2}=.38$. Significant three-way interactions of compatibility, task, and task version were observed in the latency and accuracy domains: $F$ $(2,180)=3.74, p=.03, \eta_{p}^{2}=.04$, and $F(2,180)=3.23, p=$ $.04, \eta_{p}{ }^{2}=.04$, respectively. Moreover, reaction times were faster and fewer errors were made in joint go/no-go tasks than in forced two-choice tasks, $F(1,90)=102.70, p<.01, \eta_{p}{ }^{2}=$ .53 , and $F(1,90)=139.24, p<.01, \eta_{p}{ }^{2}=.61$. Additionally,

\footnotetext{
${ }^{3}$ In the joint go/no-go conditions, we aggregated the data across each pair of participants to keep the numbers of trials in the forced two-choice (504 trials) and joint go/no-go (252 for each participant) conditions the same within each analysis of variances in this and the subsequent experiment. To check for biases due to the aggregation of pairs of participants in the joint go/no-go conditions of Experiments 2 and 3, we conducted $2 \times 3 \times 2 \times 2$ analyses of variances for each experiment in the latency as well as the accuracy domain with the factors Stimulus Color (trials requiring responses to red vs. green stimuli) and Compatibility (compatible vs. neutral vs. incompatible trials) as repeated measures and the factors Task (forced two-choice vs. joint go/no-go) and Task Version (horizontal vs. vertical) as manipulations between subjects. We expected that the SCEs of the two coacting participants in the joint go/no-go conditions would be equivalent and, hence, expected no interaction of the factors Stimulus Color and Compatibility. In summary, the data of Experiments 2 and 3 revealed comparable SCEs of each pair of participants in the horizontal and vertical joint go/no-go conditions, suggesting that the data aggregation across each pair of participants was quite appropriate. The only interaction of the factor Stimulus Color that reached significance was an interaction of stimulus color, task, and compatibility in the accuracy data of Experiment 3. This interaction was based on differences in the SCEs for red and green stimuli in the horizontal forced two-choice condition: Participants showed smaller SCEs for red stimuli $(M=5.64 \%, S D=9.20)$ than for green stimuli $(M=9.38 \%, S D=10.55), t(27)=-2.54, p<.05$. This effect emerged in neither the two joint go/no-go conditions nor the vertical forced two-choice condition. Thus, the joint go/no-go tasks were not affected by aggregating across each pair of participants.
}
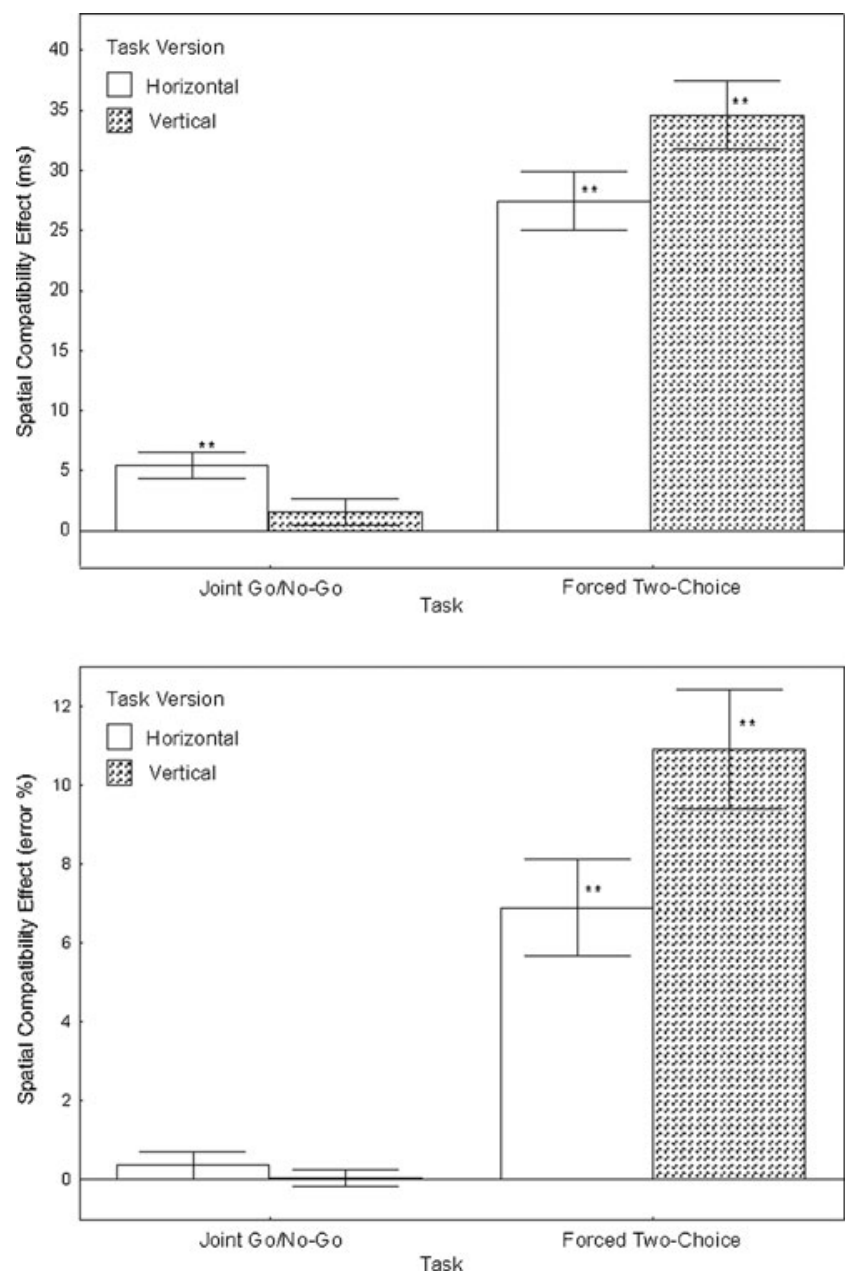

Fig. 5 Experiment 2: Spatial compatibility effects in the latency domain (upper panel) and the error domain (lower panel) as a function of task version (horizontal vs. vertical) and task (joint go/no-go vs. forced twochoice). Error bars show standard errors of the means. ${ }^{*} p<.05,{ }^{* *} p<.01$

responses were faster in the horizontal task versions, $F(1,90)=$ $31.27, p<.01, \eta_{p}{ }^{2}=.26$. Finally, task and task version interacted significantly in the latency domain, $F(1,90)=9.99, p<$ $.01, \eta_{p}{ }^{2}=.1$ : The difference in the response latencies between the tasks was larger for the vertical stimuli.

To assess the two predictions, (a) absent SCEs in the vertical joint go/no-go task and (b) SCEs in both the vertical and horizontal forced two-choice tasks, follow-up $t$ tests were conducted. (a) In the latency domain, the horizontal joint go/ no-go task showed a significant SCE, $t(22)=4.65, p<.01$. In contrast, and as predicted, the SCE was not significant in the vertical joint go/no-go task in the latency domain $(t=1.35)$. The difference between the SCEs in both the horizontal and vertical task versions was significant, $t(44)=2.32, p=.03$. There were no significant SCEs in the accuracy domain for either task 
version: In both groups, $|t| \mathrm{s}<1$ (see Fig. 5 for the means). ${ }^{4}$ (b) Significant SCEs were observed in both the horizontal forced two-choice task $[t(23)=10.91, p<.01$, and $t(23)=-6.73$, $p<.01$, for the latency and accuracy domains] and the vertical forced two-choice task $[t(23)=11.62, p<.01$, and $t(23)=-9.97$, $p<.01$, for the latency and accuracy domains]. SCEs were larger in the vertical than in the horizontal task for the accuracy domain, $t(46)=2.41, p=.02$. In the latency domain, the difference pointed in the same direction but fell short of significance, $t(46)=1.83, p=.07$.

\section{Discussion}

In Experiment 2, SCEs in joint go/no-go tasks were examined. Previous studies had repeatedly reported SCEs when two participants performed a spatial compatibility task together (e.g., Sebanz et al., 2003). Referring back to our argument, these joint SCEs were due to the fact that the horizontal spatial response dimension was emphasized by the experimental setting, which led to a stimulus-response overlap (two participants sat next to each other and responded to horizontally arranged stimuli with horizontally arranged response keys). By switching to a vertical task version and looking at SCEs contingent on the vertical dimension, we eliminated the source of interference: The social setting did not emphasize the vertical spatial dimension, and thus no stimulus-response overlap emerged (Kornblum et al., 1990). In line with our assumptions, SCEs were absent in the vertical joint go/no-go task.

At the same time, in both the horizontal and vertical forced two-choice tasks, a significant SCE was found. This

\footnotetext{
${ }^{4}$ Interestingly, we observed smaller joint SCEs in this and in the subsequent experiment (5 and $6 \mathrm{~ms}$, respectively) in comparison to the joint SCE of $11 \mathrm{~ms}$ reported in the first experiment of Sebanz et al. (2003). This difference might be due to the fact that Sebanz et al. (2003) used biological spatial stimuli - that is, a hand pointing either to the right or to the left - that might have induced "the affordance of a turn-taking signal" (Sebanz, 2004, p. 62) and increased the joint SCEs. Natalie Sebanz (2004) actually tested this alternative account in Experiment $5 \mathrm{a}$ of her dissertation, using arrows as abstract stimuli that pointed either to the left or to the right. She reported a joint SCE of approximately $5 \mathrm{~ms}$ and concluded that "The fact that the joint compatibility effect was smaller for arrows compared to pointing fingers suggests that the joint compatibility effect may be modulated by the nature of the pointing stimuli" (Sebanz, 2004, pp. 65-66). Sebanz could increase the joint SCE for abstract stimuli up to $15 \mathrm{~ms}$ in Experiment $5 \mathrm{~b}$ when presenting the stimuli clearly on the left- or the right-hand side of the screen, making the spatial dimension very salient (whereas our stimuli were presented closer to the middle of the screen). Tsai et al. (2006) also observed a larger joint SCE (15.2 ms), despite the fact that we used the same stimuli. This might be due to the fact that our participants were motivated to respond very quickly in the joint conditions of Experiments 2 and 3, so that the size of joint SCEs was diminished. We observed mean response latencies of 288 and $301 \mathrm{~ms}$, respectively, whereas Tsai et al. (2006) reported a mean response latency of $442 \mathrm{~ms}$ in the joint go/no-go condition.
}

dispels the possibility that the vertical task simply did not produce SCEs in the first place and is inconsistent with the view that shared task representations cause joint SCEs. If task representations had been functionally equivalent in the forced two-choice and the joint go/no-go tasks, then SCEs would have been observed in the vertical joint go/no-go task as well.

The present experiment provides evidence for the hypothesis that SCEs in joint go/no-go tasks are caused by the salience of a spatial response dimension and not by shared task representations. To ensure that the conditions were as similar as possible to those in the social Simon studies reported by others (e.g., Sebanz et al., 2003), participants in the joint go/no-go conditions of Experiment 2 responded on separate response pads. However, the missing joint SCE in the vertical condition might also be attributed to a distant arm position of both coacting participants. As was pointed out in the introduction, Guagnano et al. (2010) provided evidence that the seating distance of two persons performing joint go/no-go tasks determines the occurrence of joint SCEs. They demonstrated joint SCEs only in conditions in which participants sat within arm's reach and not when they were sitting farther apart. To rule out the possibility that the absent joint $\mathrm{SCE}$ was due to arm positions being too far apart in the vertical joint go/no-go task, a third experiment was conducted that differed with regard to the response key arrangements, and thus implemented closer arm positions of the coacting participants in the joint go/ no-go tasks. Specifically, the same response device was used in all conditions.

By using the same response device, we attempted to rule out another possible confound in Experiment 2: It might be questioned whether the conditions of Experiment 2 were procedurally comparable, since different response devices were used across the conditions (see Fig. 4). It might be argued that the differences in the response arrangements specifically covaried with the reported effects of the vertical task version. As is shown in Fig. 4, participants performing the vertical task version either responded on a single response pad with two vertically arranged response keys (forced two-choice task) or had to respond on one of two horizontally arranged response pads, each consisting of two vertically arranged response keys (joint go/no-go task). Thus, in the joint go/no-go task, the response arrangements included not only the vertical, but also the horizontal dimension. That, in turn, might have degraded the joint SCE in this condition. Therefore, it might be questioned whether the SCE would increase in the vertical joint go/no-go task if both participants had to respond on the same response pad containing two vertically arranged keys. By conceptually replicating Experiment 2 and using the same response pad in all conditions, this possible confound could be removed. 
Additionally, finding a result pattern similar to that of Experiment 2 would also demonstrate that the results are replicable and invariant across the specific response devices used in the present experiments.

\section{Experiment 3: Joint go/no-go tasks with identical response devices}

In Experiment 3, we aimed to replicate the result pattern of Experiment 2 while controlling for the response device. Again, participants performed the vertical or the horizontal task version; they performed the task either alone (forced two-choice task) or together with another participant (joint go/no-go task). Analogously to the assumptions and results found in Experiment 2, (a) SCEs should be absent in the vertical joint go/no-go task, since the seating alignment did not emphasize the vertical spatial response dimension, whereas in the horizontal joint go/no-go task, we expected to find SCEs. In addition, (b) SCEs should be observed in the vertical and horizontal forced two-choice tasks. A small but important difference between Experiments 2 and 3 was the control of response devices: In Experiment 3, participants in all conditions responded on the same response pad. The only difference between the horizontal and vertical task conditions consisted of either a vertical or horizontal response key arrangement (see note 2). In using only one response pad, coacting participants had to place their arms closely together. Thus, we were able to examine the role of arm position for joint SCEs, and specifically, we were able to rule out that the joint SCE in the vertical joint go/no-go task of Experiment 2 was absent due to the too-distant arm positions of the coacting participants (see Guagnano et al., 2010).

\section{Method}

The procedure and design were identical to those of Experiment 2, except for the differences indicated below.

Participants A group of 168 right-handed University of Freiburg students with different majors participated for course credit or as paid volunteers; their mean age was 23 $(S D=3.4)$, ranging from 18 to 41 years. All participants had normal or corrected-to-normal vision and were randomly assigned to one of four experimental conditions defined by task version (horizontal vs. vertical) and task (forced two-choice vs. joint go/no-go). The forced two-choice conditions comprised 56 participants, and the joint conditions included 56 pairs of participants. Two pairs of participants in the joint go/no-go conditions were excluded from the analyses because they each responded to $26 \%$ of the no-go stimuli.
Materials and procedures The only difference between Experiments 2 and 3 consisted of the different response devices. In all conditions of Experiment 3, participants responded on a single response pad with two response keys that were positioned in the middle in front of the PC screen. In the horizontal task, the response keys were horizontally arranged; in the vertical task, they were vertically arranged. The participants performing the forced two-choice tasks pressed either the upper and lower or the right and left key to respond to red and green circles. Each participant performing the joint go/no-go task operated one of the two response keys. In the horizontal joint go/no-go task, the participant seated on the left always pressed the left key, while the participant seated on the right always pressed the right key. In the vertical joint go/no-go task, the participant sitting to the right operated one key (e.g., the upper key), whereas the participant sitting to the left pressed the other key (e.g., the lower key). Whether the participant seated on the right or left side pressed the lower or upper key was counterbalanced; in the vertical forced two-choice task, it was also counterbalanced whether participants were to press the lower or the upper key with their right or left index finger. The mapping of the colors to the response key assignments was counterbalanced for all conditions. The stimulus arrangements, seating alignments, and response alignments are depicted in Fig. 6.

\section{Results}

Response latencies were preprocessed as in Experiment 1, which excluded $3.70 \%$ of the trials. The mean response latencies and mean error rates for compatible, incompatible, and neutral trials are presented in Table 3 of the Appendix, separately for the forced two-choice tasks and the joint go/ no-go tasks. In Fig. 7, SCEs are presented as a function of task version (horizontal vs. vertical) and task (joint go/no-go vs. forced two-choice) for latencies (upper panel) and error rates (lower panel).

Response latencies and the accuracy data were submitted to analyses of variance with the factors Compatibility (compatible vs. incompatible vs. neutral) $\times$ Task Version (horizontal vs. vertical) $\times$ Task (joint go/no-go vs. forced twochoice) and repeated measures on the first factor (see note 3 ). These analyses revealed main effects of compatibility in the latency domain, $F(2,212)=250.67, p<.01, \eta_{p}{ }^{2}=.70$, and the accuracy domain, $F(2,212)=57.44, p<.01, \eta_{p}{ }^{2}=$ .35: Faster responses were given and fewer errors were made in compatible than in incompatible trials. Significant interactions of compatibility and task for the response latencies, $F(2,212)=119.06, p<.01, \eta_{p}{ }^{2}=.53$, and accuracy data, $F(2,212)=42.00, p<.01, \eta_{p}{ }^{2}=.28$, indicated larger SCEs in the forced two-choice tasks than in the joint go/nogo tasks. Moreover, compatibility effects were larger in the 
Fig. 6 Stimulus arrangements, participants' seating alignments, and response devices used in Experiment 3. Participants performed either a forced two-choice task (a) or a joint go/no-go task (b). Orthogonally, they performed either a horizontal task version (c) or a vertical task version (d). Participants performing the horizontal task version responded with horizontally oriented response keys, and participants performing the vertical task version responded with vertically oriented response keys. Distance information is indicated in centimeters

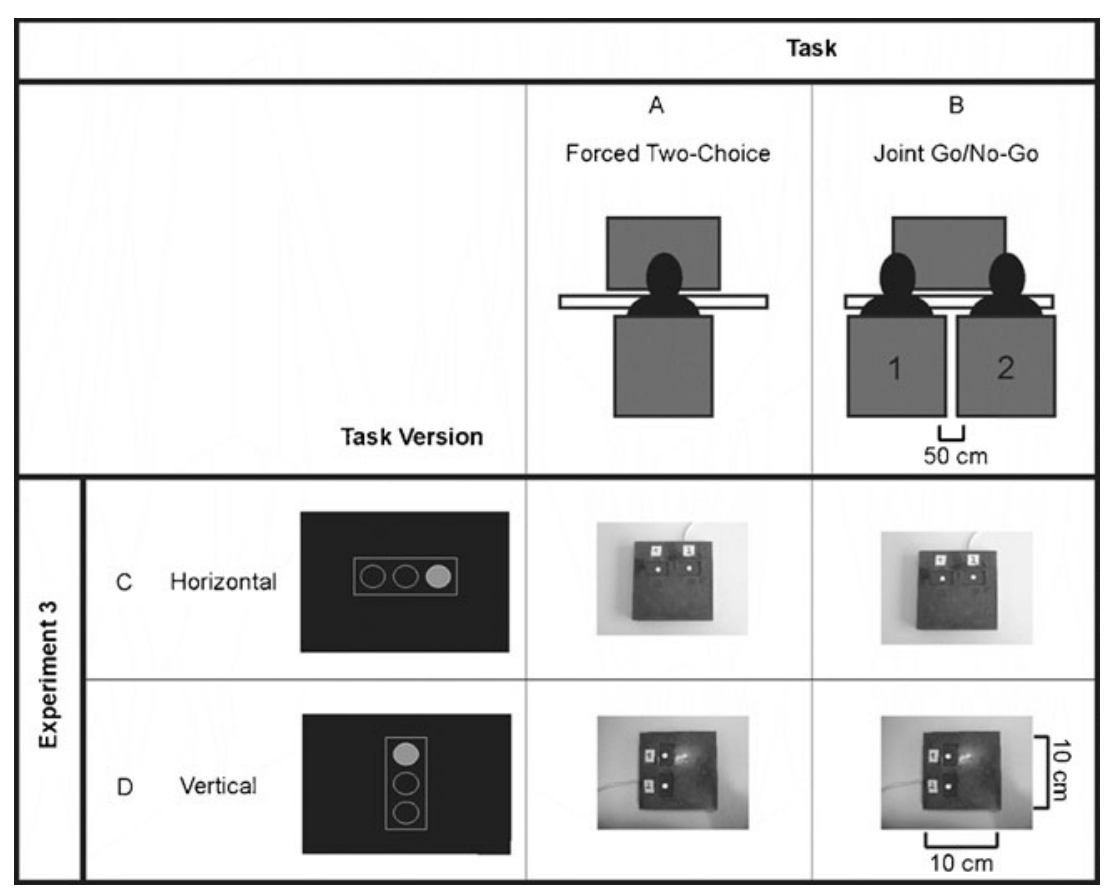

horizontal tasks than in the vertical tasks, as indicated by a significant interaction of compatibility and task version for response latencies, $F(2,212)=10.90, p<.01, \eta_{p}{ }^{2}=.09$. Overall, reaction times were faster and fewer errors were made in the joint go/no-go tasks than in the forced two-choice tasks, as indicated by a main effect of task, $\mathrm{F}(1,106)=118.40$, $p<.01, \eta_{p}{ }^{2}=.53$, and $F(1,106)=240.15, p<.01, \eta_{p}{ }^{2}=.69$, respectively. A main effect of task version in the accuracy domain, $F(1,106)=6.14, p=.02, \eta_{p}{ }^{2}=.06$, indicated that fewer errors were made in the vertical tasks.

Follow-up $t$ tests were conducted to assess the two predictions: (a) absent SCEs in the vertical joint go/no-go task and (b) SCEs in both the vertical and the horizontal forced twochoice tasks. (a) In the latency domain, the horizontal joint go/ no-go task showed a significant SCE, $t(25)=5.47, p<.01$. Replicating the results of Experiment 2, the SCE in the latency domain was not significant in the vertical joint go/no-go task, $t=$ 1.24. The difference between the SCEs in the horizontal and vertical joint go/no-go task versions was significant, $t(52)=$ $2.63, p=.01$. For either task version, there were no significant SCEs in the accuracy domain (all $|t| \mathbf{S}<1.3$; see Fig. 7 for the means). (b) As predicted, significant SCEs were observed both in the horizontal forced two-choice task $[t(27)=13.19, p<$ .01 , and $t(27)=-5.32, p<.01$, for the latency and accuracy domains], and in the vertical forced two-choice task [t(27) = $12.24, p<.01$, and $t(27)=-8.64, p<.01$, for the latency and accuracy domains]. The SCE was larger in the horizontal than in the vertical task for the latency domain, $t(54)=$ $3.37, p=.01$, while this difference was not significant in the accuracy data, $t=0.77$.

\section{Discussion}

In Experiment 3, the procedure of Experiment 2 was replicated, except that participants responded on a single response pad that was identical in the vertical and horizontal task versions (only the orientation of the response keys differed). The results for joint SCEs found in Experiment 2 were replicated. Importantly, the joint SCEs of Experiments 2 and 3 did not significantly differ in size in either the vertical joint go/no-go task (Exp. $2, M=1.59, S D=5.63$; Exp. 3, $M=1.59, S D=6.76 ; t=-0.01)$ or the horizontal joint go/no-go task (Exp. 2, $M=5.43, S D=5.61$; Exp. 3, $M=$ $6.08, S D=5.67 ; t=-0.40)$. In demonstrating that SCEs were absent not only in the vertical joint go/no-go task of Experiment 2, but also in Experiment 3, we were able to rule out that the joint $\mathrm{SCE}$ in the vertical task version of Experiment 2 was absent because the arm positions of the coacting participants were too distant. By using the identical response device in all conditions, we were also able to rule out that the use of different response devices led to the absence of an SCE in the vertical joint go/no-go task of Experiment 2.

Although the joint SCEs were comparable in size in Experiments 2 and 3, SCEs in the vertical and horizontal forced two-choice tasks differed between the experiments. In Experiment 2, the SCE in the forced two-choice vertical task was larger than in Experiment 3, whereas the opposite was observed for SCEs in the horizontal forced two-choice tasks (see Figs. 5 and 7 for the means). This difference is difficult to explain, but it should be noted that the forced 

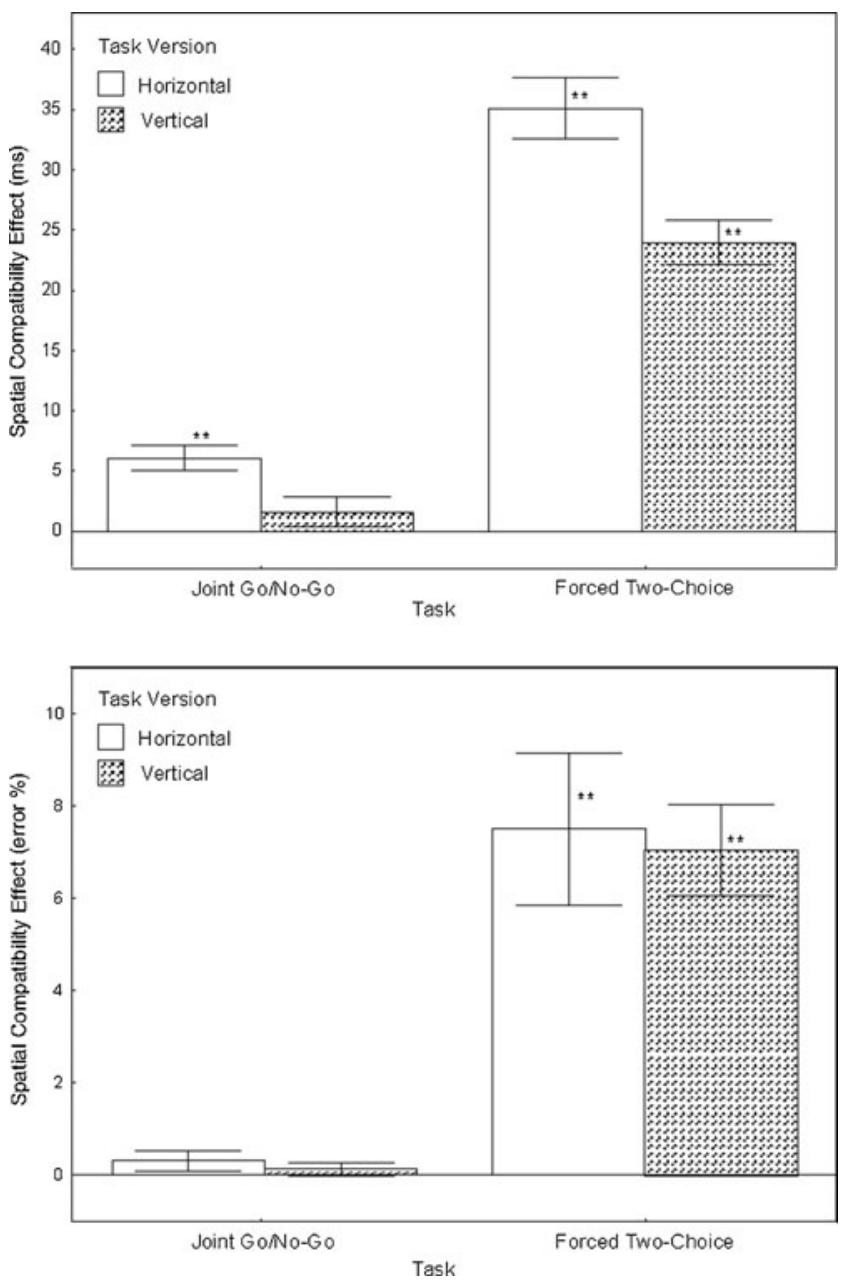

Fig. 7 Experiment 3: Spatial compatibility effects in the latency domain (upper panel) and the error domain (lower panel) as a function of task version (horizontal vs. vertical) and task (joint go/no-go vs. forced twochoice). Error bars show standard errors of the means. ${ }^{*} p<.05,{ }^{* *} p<.01$

two-choice conditions served primarily as baseline conditions, to ensure that reliable SCEs occurred in both forced two-choice conditions.

\section{General discussion}

In Experiment 1, SCEs in individual go/no-go tasks were examined. As expected, SCEs were found in individual go/ no-go tasks only when the participants responded with a left or a right joystick movement; replicating previous results, SCEs were not observed when the participants responded with left or right keypresses. In Experiments 2 and 3, SCEs in joint go/no-go tasks were examined. As expected, SCEs were found in joint go/no-go tasks only with horizontally, not with vertically, arranged stimuli. Experiments 2 and 3 revealed a similar result pattern, although different response devices were used in both experiments. All of the experiments revealed reliable SCEs in forced two-choice conditions.

In demonstrating SCEs in joint but not in individual go/ no-go tasks (e.g., Sebanz et al., 2003), joint SCEs had typically been explained in terms of shared task representations: The action that one performed and the action that one observed were assumed to be represented in functionally equivalent ways (see, e.g., Hommel et al., 2001; Prinz, 1997). In the present work, we attempted to provide evidence for an alternative explanation of joint SCEs - an account in terms of spatial response coding. Specifically, we assumed that joint go/no-go tasks render the spatial response dimension more salient than do individual go/no-go tasks, because the task setting induces participants to perceive themselves as being the left or the right participant. This in turn induces participants to code their responses spatially, leading to a stimulus-response overlap that is similar to the stimulus-response overlap that is assumed to cause SCEs in forced two-choice tasks (Kornblum et al., 1990).

Our results provide evidence that SCEs are observed whenever the task setting induces participants to code their responses spatially. By emphasizing and deemphasizing the spatial response dimension in Experiments 1-3, SCEs were switched on and off in line with our assumptions. In the present Experiment 1, the salience of the spatial response dimension was increased by requiring either left or right joystick movements as responses instead of keypresses. In line with our assumption, an SCE was found in the individual go/no-go joystick group. In Experiments 2 and 3, we deemphasized the spatial response dimension in a vertical joint go/no-go task, a condition in which joint SCEs would normally have been expected (i.e., two persons worked together on a joint go/no-go task, and should thus have formed co-representations of the task; e.g., Sebanz et al., 2003, 2005). An SCE was found in joint go/no-go tasks only if the (horizontal) response dimension was emphasized by a corresponding (horizontal) seating alignment. In the vertical joint go/no-go task, the participant seating positions presumably increased the salience of the horizontal response dimension, but here, the horizontal response dimension did not overlap with the vertical stimulus dimension. In line with this assumption, joint SCEs were not found in the vertical joint go/no-go conditions of Experiments 2 and 3. In Experiment 3, participants responded on the same response pad in joint go/no-go conditions, and still no joint SCE was found for the vertical task. Thus, the missing joint SCE in the vertical joint go/no-go task of Experiment 2 cannot be explained by too-distant arm positions (Guagnano et al., 2010; see below). The account of shared task representations gives little reason to doubt that participants should form shared task representations irrespective of the stimulus and response key arrangements used in Experiments 2 and 3. Thus, 
joint SCEs should have prevailed, irrespective of the stimulus arrangement. However, as we did not find joint SCEs in the vertical joint go/no-go task, our data did not confirm the predictions made by the account of shared task representations.

Our spatial-response-coding account of joint SCEs is in line with recent the findings of Guagnano et al. (2010) and Dolk et al. (2011) that have demonstrated the relevance of a spatial component for joint SCEs. The present research, however, provides more conclusive and direct evidence for this alternative explanation. First, we were able to directly disentangle effects based on spatial components, on the one hand, and shared task representations, on the other hand, while we relied as closely as possible on the procedure reported in the seminal work of Sebanz et al. (2003). Note that both Guagnano et al. and Dolk et al. introduced further influencing factors in order to examine the role of the spatial dimension in joint go/no-go tasks (e.g., seating distance, crossed-hand effect, or proprioceptive events). Moreover, both studies used spatial compatibility tasks rather divergent from those of the original studies (e.g., with acoustic stimuli or simultaneous stimulus presentation). Second, and more importantly, by examining the role of the spatial response dimension in an individual go/no-go task as well, we were able to generalize the effect and to address the two lines of Sebanz et al.'s (2003) original argument comprehensively. Our observation of SCEs in individual go/no-go tasks when participants responded with a joystick movement demonstrated that it is not simply the coactor as a reference point (Guagnano et al., 2010) or an irregular and attention-attracting event (Dolk et al., 2011) that modulate SCEs and specifically leads to SCEs in a joint task setting. Instead, we demonstrated more generally that each increase in the salience of the spatial response dimension induces a spatial response coding that finally leads to a stimulus-response overlap (Kornblum et al., 1990) - irrespective of the specific task settings. Therefore, according to our point of view, a spatial-responsecoding account can explain joint SCEs more comprehensively and parsimoniously.

Interestingly, SCEs have often been found to be smaller in joint go/no-go tasks than in forced two-choice tasks (e.g., Welsh, 2009; Welsh, Higgins, Ray, \& Weeks, 2007) - a result pattern that was also confirmed in our studies. Guagnano et al. (2010) argued that this difference might indicate different processes underlying joint SCEs and the SCEs found in forced two-choice tasks. As we have already described in the introduction, Guagnano et al. assumed that joint SCEs are based on the facilitation of compatible trials relative to neutral or incompatible trials via response priming. However, Ferraro et al.'s (2011) study ruled out this explanation. According to our point of view, the same process underlies SCEs in forced two-choice tasks and joint go/no-go tasks-namely, a stimulus-response overlap. Note that already Sebanz et al. (2003) had proposed a functional similarity between the SCEs of forced two-choice and joint go/no-go tasks that is also based on a stimulus-response overlap. However, whereas Sebanz et al. (2003) assumed shared task representations to be the crucial factor, we suggest that the stimulus-response overlap in joint task settings is due to an increased spatial salience that allows participants to code their responses spatially. Smaller SCEs in joint go/no-go tasks might be explained by the assumption that the spatial response dimension is most salient in a forced two-choice task, in which participants are responsible for both the left and right response keys. Factors that strengthen the salience of the spatial response dimension might work additively. ${ }^{5}$ Indirect evidence for this assumption is provided in the present Experiment 1, in which the largest SCE was found in the forced two-choice joystick condition (a forced two-choice setting and the joystick movements might additively strengthen the salience of the spatial response dimension).

Inspecting the mean reaction times for the different stimulus types in Experiments 1-3 (see the tables in the Appendix), we found accelerated reaction times for neutral stimuli relative to compatible stimuli in individual and joint go/no-go tasks and smaller facilitation than interference effects in the forced two-choice conditions. A similar result pattern has also been found in other studies examining SCEs, the most relevant for our studies being the study of Sebanz et al. (2003). Umiltà, Rubichi, and Nicoletti (1999) examined the sizes of the interference and facilitation effects in spatial compatibility tasks. They argued that responses are faster and more accurate for neutral stimuli because the neutral position is favored by visual acuity in a standard spatial compatibility task. In manipulating the positions of neutral stimuli, they found interference and facilitation effects of similar sizes. Presumably, a processing advantage for neutral stimuli might also explain the present result pattern regarding accelerated responses to neutral stimuli. Given the possibility of a processing advantage for neutral stimuli, the present data might not conclusively answer the question of whether the SCEs found in the present go/no-go tasks were caused by interference or facilitation effects (but note that the results of Ferraro et al., 2011, might suggest that the SCEs in the present go/no-go tasks were caused by interference rather than by facilitation effects).

As noted earlier, Guagnano et al. (2010) demonstrated an influence of participants' seating distance on joint SCEs: For participants sitting beyond arm's reach, no joint SCEs were

\footnotetext{
${ }^{5}$ We thank an anonymous reviewer for pointing out this possibility
} 
observed, while for participants sitting within arm's reach, SCEs reemerged. Evidence for an influence of participants' arm positions on the size of joint SCEs was not found in the present experiments. Because participants responded on a single response pad in the joint go/no-go conditions of Experiment 3, one might suggest that joint SCEs should have been larger than the joint SCEs of Experiment 2, in which participants responded on separate response pads (since the arm positions of both coacting participants were closer together when they responded on the same response pad). Instead, the joint SCEs of Experiments 2 and 3 were comparable in size in both the vertical and the horizontal joint go/no-go tasks. One possible interpretation of this finding is that joint SCEs do not decrease linearly with increasing seating distance, but increasing seating distance might lead to a qualitative change from joint SCE to no joint SCE at a certain distance point. This is in line with the assumption that the coacting participant is either taken as a spatial reference point (within arm's reach) or not (outside arm's reach), depending on whether or not the coacting person is perceived as being an integral part of the task (see Guagnano et al., 2010, for a similar argument). Note, however, that the arms of the coacting participants were relatively close together, even in our Experiment 2 (the seating distance in our study was about $50 \mathrm{~cm}$, while participants sat $1.5 \mathrm{~m}$ away from each other in the study of Guagnano et al., 2010). Future research will have to investigate in more detail whether arm position changes joint SCEs qualitatively (switches joint SCEs on or off) or quantitatively (gradually increasing joint SCEs with decreasing seating distance).

Although the present research indicates that joint SCEs might be mainly driven by spatial response coding, published joint action research has nevertheless suggested that social components influence joint SCEs. These results do not necessarily contradict a spatial response-coding account of joint SCEs, as we will explain next. Tsai, Kuo, Hung, and Tzeng (2008), for example, found joint SCEs although the coactor sat in another room (but see Welsh et al., 2007, for a different result pattern). On the face of it, it seems implausible that spatial response coding could explain the result pattern of Tsai et al. (2008), because no coactor was present, and thus the spatial response dimension was not highlighted. However, a closer look at the procedure of Tsai et al. (2008) suggests that the experiment might still have reinforced spatial response coding. For instance, Tsai et al. (2008) strengthened participants' perception of a coacting task setting in advance of the experiment by asking pairs of participants to practice the task in a training phase in which actual interaction was involved. Additionally, the participants were explicitly told that their coactor would press a left response key, while they should press a right response key. According to a spatial-responsecoding account, training phase or mere instruction of one's own response as the right response and of the coactor's response as the left response might have allowed participants to code their responses spatially. Moreover, Tsai and Brass (2007) found that SCEs emerged only when participants performed a joint go/no-go task with an image of a human hand but not with an image of a wooden hand ("Pinocchio's hand"). Interestingly, Müller, Brass, et al. (2011) demonstrated joint SCEs even with an image of a wooden hand, but only when the animacy of Pinocchio was increased (participants watched a video of Pinocchio and had to take the perspective of Pinocchio for a certain amount of time). Müller, Brass, et al. suggested as one hypothesis that only the actions of similar others are co-represented (see also Müller, Kühn, et al., 2011). Reinterpreting the results of Müller, Brass, et al. and Tsai and Brass, it might be possible that social components influence the ease of forming spatial response codes: Routinely, participants might only use similar others - that is, other biological agents - as spatial reference points. Similar others are potentially relevant for oneself, while nonhumans or outgroup members might not be sufficiently salient to serve as reference points, or might even be perceived as potentially distracting (and thus participants might actively narrow their attentional focus to exclude them). Other studies have also demonstrated an influence of social factors on joint SCEs. Those effects might be explained similarly: Hommel et al. (2009) found joint SCEs only when the coactor (who was in fact a confederate) was friendly, but not when the confederate showed unfriendly behavior. A coactor might only serve as a spatial reference point for spatial response coding when being perceived as a friendly, cooperative person (see also Ruys \& Aarts, 2010).

Thus, social factors might indeed influence joint SCEs (as was also suggested by Wenke et al., 2011, who focused on the persons acting in joint go/no-go tasks in their actor co-representation account; see also Philipp \& Prinz, 2010). But this influence might be mediated by a spatial response coding: A subject or object included in the experimental setting might be taken as a spatial reference point of one's own response if the subject or object is perceived as being an integral part of the task. Participants thus code their responses as left or right, and we argue that this spatial response coding creates a source of interference, because the response dimension now overlaps with the stimulus dimensions. This influence does not need to be mediated by shared task representations in joint go/no-go tasks. At least, our experiments, as well as the experiments provided by Guagnano et al. (2010) and Dolk et al. (2011), do not suggest this.

Although our results do not support a shared-taskrepresentation account of joint SCEs, joint interference effects have also been demonstrated for the SNARC effect (Atmaca, Sebanz, Prinz, \& Knoblich, 2008) and for joint flanker effects (Atmaca, Sebanz, \& Knoblich, 2011). The SNARC effect also relies on spatial numerical associations. Thus, the spatial dimension of the task and the spatial 
dimension of the experimental setting might be confounded in a joint SNARC effect as well. The flanker task, however, does not include a spatial dimension and might provide an unconfounded measurement of joint interference effects.

Our experiments suggest that the typical result pattern defining social Simon effects-(a) SCEs in forced twochoice tasks, but no SCEs in individual go/no-go tasks, and (b) SCEs in joint go/no-go tasks - is confounded with differences in the salience of the spatial response dimension between individual go/no-go tasks and joint go/no-go tasks. By removing this confound and manipulating the social setting and the salience of the spatial response dimension orthogonally, we found that the absence versus presence of SCEs depended on spatial response coding and was not a function of the social setting. Due to the confound of the social setting and the salience of the spatial response dimension, future research on socially shared task representations might profit from developing and using interference paradigms in which another dimension, other than the spatial dimension, mediates interference effects. Then, the social setting would be responsible for social compatibility effects, and not the interference task itself.

Author note The research reported in this article was supported by Grant No. Kl 614/31-1 from the Deutsche Forschungsgemeinschaft to C. Klauer. We thank Sven-Erik Wulf for helping us with the materials used in Experiments 2 and 3.

\section{Appendix}

Table 1 Experiment 1: Mean response latencies (in milliseconds), error rates (as percentages), and standard deviations for compatible, neutral, and incompatible trials as a function of task and response device

${ }^{\text {a }} \mathrm{SCE}$, spatial compatibility effect.

Table 2 Experiment 2: Mean response latencies (in milliseconds), error rates (as percentages), and standard deviations for compatible, neutral, and incompatible trials as a function of task and task version

${ }^{a}$ SCE, spatial compatibility effect.

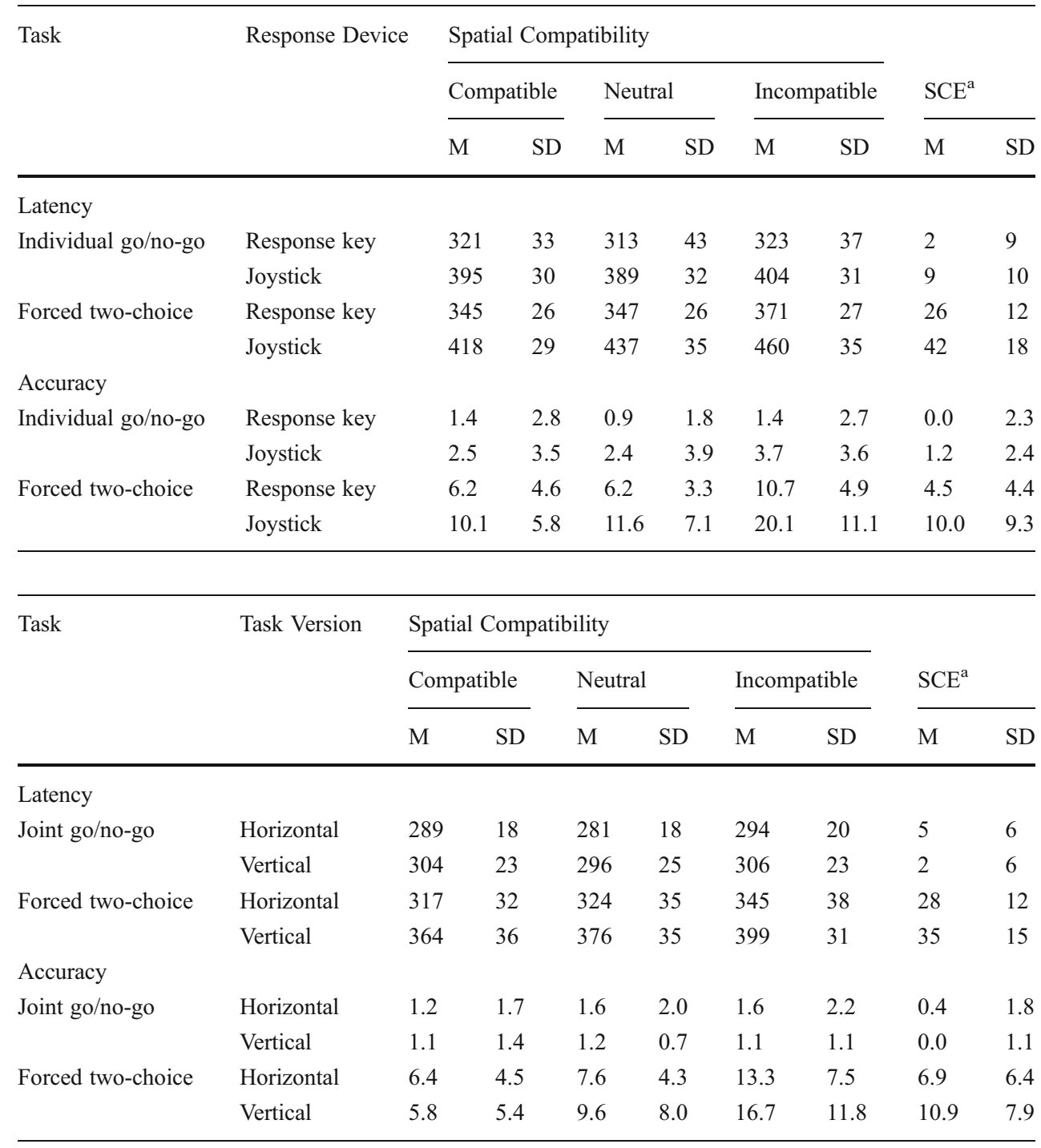


Table 3 Experiment 3: Mean response latencies (in milliseconds), error rates (as percentages), and standard deviations for compatible, neutral, and incompatible trials as a function of task and task version

${ }^{\mathrm{a}} \mathrm{SCE}$, spatial compatibility effect.

\begin{tabular}{|c|c|c|c|c|c|c|c|c|c|}
\hline \multirow[t]{3}{*}{ Task } & \multirow[t]{3}{*}{ Task Version } & \multicolumn{6}{|c|}{ Spatial Compatibility } & & \\
\hline & & \multicolumn{2}{|c|}{ Compatible } & \multicolumn{2}{|c|}{ Neutral } & \multicolumn{2}{|c|}{ Incompatible } & \multicolumn{2}{|c|}{$\mathrm{SCE}^{\mathrm{a}}$} \\
\hline & & M & SD & M & $\mathrm{SD}$ & M & SD & $\mathrm{M}$ & SD \\
\hline \multicolumn{10}{|l|}{ Latency } \\
\hline \multirow[t]{2}{*}{ Joint go/no-go } & Horizontal & 301 & 27 & 294 & 30 & 307 & 27 & 6 & 6 \\
\hline & Vertical & 305 & 23 & 298 & 23 & 307 & 23 & 2 & 7 \\
\hline \multirow[t]{2}{*}{ Forced two-choice } & Horizontal & 342 & 31 & 354 & 33 & 377 & 33 & 35 & 14 \\
\hline & Vertical & 347 & 24 & 353 & 24 & 371 & 24 & 24 & 10 \\
\hline \multicolumn{10}{|l|}{ Accuracy } \\
\hline \multirow[t]{2}{*}{ Joint go/no-go } & Horizontal & 0.7 & 0.7 & 0.8 & 1.0 & 1.0 & 1.0 & 0.3 & 1.1 \\
\hline & Vertical & 0.5 & 0.6 & 0.9 & 1.1 & 0.6 & 0.7 & 0.1 & 0.8 \\
\hline \multirow[t]{2}{*}{ Forced two-choice } & Horizontal & 6.8 & 4.4 & 8.4 & 6.4 & 14.3 & 10.8 & 7.5 & 9.2 \\
\hline & Vertical & 4.1 & 3.1 & 5.7 & 3.8 & 11.2 & 6.7 & 7.1 & 5.5 \\
\hline
\end{tabular}

\section{References}

Atmaca, S., Sebanz, N., \& Knoblich, G. (2011). The joint flanker effect: Sharing tasks with real and imagined co-actors. Experimental Brain Research, 211, 371-385. doi:10.1007/s00221-011-2709-9

Atmaca, S., Sebanz, N., Prinz, W., \& Knoblich, G. (2008). Action corepresentation: The joint SNARC effect. Social Neuroscience, 3, 410-420. doi:10.1080/17470910801900908

Botvinick, M., \& Cohen, J. (1998). Rubber hands "feel" touch that eyes see. Nature, 391, 756. doi:10.1038/35784

Clark, H. H. (1996). Using language. Cambridge, U.K.: Cambridge University Press.

Clark-Carter, D. (2004). Quantitative psychological research: A student's handbook. New York, NY: Psychology Press.

Dittrich, K., \& Klauer, K. C. (2012). Does ignoring lead to worse evaluations? A new explanation of the stimulus devaluation effect. Cognition \& Emotion, 26, 193-208. doi:10.1080/ 02699931.2011 .570313

Dolk, T., Hommel, B., Colzato, L. S., Schütz-Bosbach, S., Prinz, W., \& Liepelt, R. (2011). How "social" is the social Simon effect? Frontiers in Psychology, 2, 1-9. doi:10.3389/fpsyg.2011.00084

Eder, A. B., \& Rothermund, K. (2008). When do motor behaviors (mis) match affective stimuli? An evaluative coding view of approach and avoidance reactions. Journal of Experimental Psychology. General, 137, 262-281. doi:10.1037/0096-3445.137.2.262

Ferraro, L., Iani, C., Mariani, M., Milanese, N., \& Rubichi, S. (2011). Facilitation and interference components in the joint Simon task. Experimental Brain Research, 211, 337-343. doi:10.1007/ s00221-011-2711-2

Greenwald, A. G. (1970). Sensory feedback mechanisms in performance control: With special reference to the ideo-motor mechanism. Psychological Review, 77, 73-99. doi:10.1037/h0028689

Guagnano, D., Rusconi, E., \& Umiltà, C. A. (2010). Sharing a task or sharing space? On the effect of the confederate in action coding in a detection task. Cognition, 114, 348-355. doi:10.1016/ j.cognition.2009.10.008

Hommel, B. (1996). S-R compatibility effects without response uncertainty. Quarterly Journal of Experimental Psychology, 49A, 546-571. doi:10.1080/027249896392496

Hommel, B., Colzato, L. S., \& van den Wildenberg, W. P. M. (2009). How social are task representations? Psychological Science, 20, 794-798. doi:10.1111/j.1467-9280.2009.02367.x
Hommel, B., Müsseler, J., Aschersleben, G., \& Prinz, W. (2001). The theory of event coding (TEC): A framework for perception and action planning. The Behavioral and Brain Sciences, 24, 849937. doi: $10.1017 / \mathrm{S} 0140525 \mathrm{X} 01000103$

Kornblum, S., Hasbroucq, T., \& Osman, A. (1990). Dimensional overlap: Cognitive basis for stimulus-response compatibility-A model and taxonomy. Psychological Review, 97, 253-270. doi:10.1037/0033-295X.97.2.253

Liepelt, R., Wenke, D., Fischer, R., \& Prinz, W. (2011). Trial-to-trial sequential dependencies in a social and non-social Simon task. Psychological Research, 75, 366-375. doi:10.1007/s00426-010-0314-3

Lu, C.-H., \& Proctor, R. W. (1995). The influence of irrelevant location information on performance: A review of the Simon and spatial Stroop effects. Psychonomic Bulletin \& Review, 2, 174-207. doi:10.3758/BF03210959

Müller, B. C. N., Brass, M., Kühn, S., Tsai, C.-C., Nieuwboer, W., Dijksterhuis, A., \& van Baaren, R. B. (2011a). When Pinocchio acts like a human, a wooden hand becomes embodied. Action corepresentation for non-biological agents. Neuropsychologia, 49, 1373-1377. doi:10.1016/j.neuropsychologia.2011.01.022

Müller, B. C. N., Kühn, S., van Baaren, R. B., Dotsch, R., Brass, M., \& Dijksterhuis, A. (2011b). Perspective taking eliminates differences in co-representation of out-group members' actions. Experimental Brain Research, 211, 423-428. doi:10.1007/s00221-011-2654-7

Philipp, A. M., \& Prinz, W. (2010). Evidence for a role of the responding agent in the joint compatibility effect. Quarterly Journal of Experimental Psychology, 63, 2159-2171. doi:10.1080/ 17470211003802426

Prinz, W. (1997). Perception and action planning. European Journal of Cognitive Psychology, 9, 129-154. doi:10.1080/713752551

Ruys, K. I., \& Aarts, H. (2010). When competition merges people's behavior: Interdependency activates shared action representations. Journal of Experimental Social Psychology, 46, 11301133. doi:10.1016/j.jesp.2010.05.016

Sebanz, N. (2004). You are always on my mind: Representing others' actions and intentions. Berlin: Logos-Verlag.

Sebanz, N., Knoblich, G., \& Prinz, W. (2003). Representing others' actions: Just like one's own? Cognition, 88, B11-B21. doi:10.1016/S0010-0277(03)00043-X

Sebanz, N., Knoblich, G., \& Prinz, W. (2005). How two share a task: Corepresenting stimulus-response mappings. Journal of Experimental Psychology. Human Perception and Performance, 31, 1234-1246. doi:10.1037/0096-1523.31.6.1234 
Sebanz, N., Knoblich, G., Prinz, W., \& Wascher, E. (2006). Twin peaks: An ERP study of action planning and control in coacting individuals. Journal of Cognitive Neuroscience, 18, 859-870. doi:10.1162/jocn.2006.18.5.859

Shiu, L. P., \& Kornblum, S. (1999). Stimulus-response compatibility effects in go-no-go tasks: A dimensional overlap account. Perception \& Psychophysics, 61, 1613-1623. doi:10.3758/ BF03213122

Simon, J. R. (1990). The effects of an irrelevant directional cue on human information processing. In R. W. Proctor \& T. G. Reeve (Eds.), Stimulus-response compatibility: An integrated perspective (pp. 31-86). Amsterdam: North-Holland.

Tsai, C.-C., \& Brass, M. (2007). Does the human motor system simulate Pinocchio's actions? Coacting with a human hand versus a wooden hand in a dyadic interaction. Psychological Science, 18 , 1058-1062. doi:10.1111/j.1467-9280.2007.02025.x

Tsai, C.-C., Kuo, W.-J., Hung, D. L., \& Tzeng, O. J. L. (2008). Action co-representation is tuned to other humans. Journal of Cognitive Neuroscience, 20, 2015-2024. doi:10.1162/ jocn.2008.20144

Tsai, C.-C., Kuo, W.-J., Jing, J.-T., Hung, D. L., \& Tzeng, O. J.-L. (2006). A common coding framework in self-other interaction:
Evidence from joint action task. Experimental Brain Research, 175, 353-362. doi:10.1007/s00221-006-0557-9

Umiltà, C., Rubichi, S., \& Nicoletti, R. (1999). Facilitation and interference components in the Simon effect. Archives Italiennes de Biologie, 137, 139-149.

Voss, A., Leonhart, R., \& Stahl, C. (2007). How to make your own response boxes: A step-by-step guide for the construction of reliable and inexpensive parallel-port response pads from computer mice. Behavior Research Methods, 39, 797-801. doi:10.3758/BF03192971

Welsh, T. N. (2009). When $1+1=1$ : The unification of independent actors revealed through joint Simon effects in crossed and uncrossed effector conditions. Human Movement Science, 28, 726-737. doi:10.1016/j.humov.2009.07.006

Welsh, T. N., Higgins, L., Ray, M., \& Weeks, D. J. (2007). Seeing vs. believing: Is believing sufficient to activate the processes of response co-representation? Human Movement Science, 26 , 853-866. doi:10.1016/j.humov.2007.06.003

Wenke, D., Atmaca, S., Holländer, A., Liepelt, R., Baess, P., \& Prinz, W. (2011). What is shared in joint action? Issues of co-representation, response conflict, and agent identification. Review of Philosophy and Psychology, 2, 147-172. doi:10.1007/s13164-011-0057-0 